Pouvoir pathogène du germé isolé. - Éprouvé sur cobaye et lapin.

Cobaye. - Reçoit le 26 septembre, en injection intrapéritonéale, $1 / 2$ cc de ${ }^{2}$ culture en bouillon, succombe en 56 heures.

Lésions de péritonite généralisée, orchi-vaginalite, congestion inténse du foie, de la rate, des capsules surrénales, pas d'hypertrophie des ganglions mésentériques. Congestion des oreillettes.

L'ensemencement en bouillon et sur gélose du sang du cœur et du foie permet d'obtenir une culture pure de strepto-cocco-bacilles, morphologiquement identiques à ceux obtenus par l'ensemencement de la moelle osseuse de M3, et présentant lés mêmes caractères culturaux et les mêmes réactions biochimiques.

Lapin. -- Le 26 septembre, reçoit $1 / 2$ cc de culture en bouillon, en injection intraveineuse. Succombe le ge jour : amaigrissement, flaccidité abdominale, hypertrophie ganglionnaire (g. mésentériques), congestion intestinale, hépatique et splénique, granulations lenticulaires sur le foie.

L'ensemencement, en bouillon peptoné salé et sur gélose, du sang du cour et du contenu crémeux des granulations hépatiques révèle des éléments identiques à ceux isolés chez M3 et le cobaye.

Conclusions - Il sémble que l'on puisse identifier le germe rencontré au cocco-bacille de Malassez et Vignal (caractères biochimiques et culturaux, pouvoir pathogène), d'origine probablement aviaire. Fn effet; la pseudo-tuberculose fut, à plusieurs reprises, constatée dans un poulailler voisin.

On peut penser que les moutons se trouvaient, au moment des essais d'immunisation, en état d'infeciion pseudo-tuberculeuse latente.

La pseudo-tuberculose du mouton, affection rare, n'a pas été, à ma connaissance, signalée depuis les cas relatés en Australie par G. DE KocH (1928) et Gilruth (1934).

\title{
TRAITEMENT DE LA GRIPPE DU PORCELET PAR LES INJECTIONS INTRAMUSCULAIRES DE NOVARSENOBENZOL
}

\author{
par R. LARRAT
}

En Janvier 1944, une épizootie de grippe sévit à Saint-Louis (Sénégal), frappant notamment deux lots de porcelets de ceux à trois mois, en deux élevages différents (porcherie militaire de Dakar-Bango; poreherie de M. R...).

Les symptômes sont les suivants : hyperthermie (inconstante), inflammation conjonctivale, pâleur de la peau, anorexie, abatlement, adynamie, respiration dyspnéique et soufflante, toux, constipation au début; diarrhée par la suite. Une seule fois, rhinite avec épistaxis. 
La terminaison est presque toujours fatale, la mort survenant en trois à cinq jours.

A l'autopsie on relève l'infiltration du tissu conjonctif sous-cutané par un liquide jaunâtre, des lésions de broncho-pneumonie catarrhale, parfois de pleurite; un épanchement péricardique est observé dans deux cas. Du côté digestif une congestion généralisée (constante) du trac̈tus.

La maladie ne put être reproduite à partir des bactéries bipolaires isolées des lésions pulmonaires et du sang du cœur, éléments figurés venant compliquer le processus morbide.

L'inoculation du sang d'un malade à un porcelet sain n'a pas réussi, ce qui précise le caractère grippal de l'infection.

Traitement. - Huit malades sont traités successivement, chacun recevant une seule injection intramusculaire de 30 centigrammes de novarsenobenzol (dans 10 cc. d'eau distillée).

Sur six d'entre eux, cette injection est suivie d'une résolution rapide des symptòmes, la respiration reprenant très vite (en 24 heures) son rythme normal. Une suivie de 48 heures a été obtenue dans les deux autres cas.

Sans conclure à l'eflicacité certaine du traitement, j'ai voulu surtout attirer l'attention sur ses résultats souvent favorables. La guérison paraît d'autant mieux assurée que l'injection de novarsenobenzol est faite dans les heures suivant l'apparition de la dyspnée.

\title{
NOUVEL HOTE D'EGYPTIANNELLA PULLORUM ET MENSURATIONS \\ DE QUELQUES GLOBULES ROUGES D'OISEAUX
}

\author{
par P. RECEVEUR et M. THOMÉ
}

Aucun des auteurs que nous avons consultés (1) ne mentionne parmi les hôtes hébergeant Aegyptiannella pullorum l'existence de l'autruche (Struthio camelus, Tinné); aussi croyons-nous de quelque intérêt d'en rapporter le fait.

La découverte en a été faite de la manière suivante :

Deux jeunes autruchons de quelques semaines, capturés en brousse au mois de Janvier 1947, nous ont été apportés au Centre vaccinogène de Fort-Lamy. Là, ils ont été mis dans un poulailler où sont entretenues des volailles diverses, et où sévit à l'état endémique Aegyptiannella pullorum : c'est d'ailleurs dans cet élevage qu'a été constatée par l'un de nous pour la première fois l'existence de ce parasite au Tchad (2).

Les poulaillers abritant les volailles, abris assez précaires, sont envahis par Argas persicus, en grand nombre, malgré les divers essais de destruction entre 